\title{
Le rôle des organisations de la culture dans l'acculturation des pays en développement
}

\section{Sandrine Basilico}

\section{OpenEdition}

\section{Journals}

Édition électronique

URL : http://journals.openedition.org/communicationorganisation/3283

DOI : 10.4000/communicationorganisation.3283

ISSN : 1775-3546

Éditeur

Presses universitaires de Bordeaux

Édition imprimée

Date de publication : 2 janvier 2005

Pagination : 149-162

ISSN : $1168-5549$

Référence électronique

Sandrine Basilico, « Le rôle des organisations de la culture dans l'acculturation des pays en développement », Communication et organisation [En ligne], 26 | 2005, mis en ligne le 19 juin 2012, consulté le 19 avril 2019. URL : http://journals.openedition.org/communicationorganisation/3283 DOI : 10.4000/communicationorganisation.3283

Ce document a été généré automatiquement le 19 avril 2019

(c) Presses universitaires de Bordeaux 


\title{
Le rôle des organisations de la culture dans l'acculturation des pays en développement
}

\author{
Sandrine Basilico
}

L'expression « mondialisation de la culture » désigne la circulation de produits culturels à l'échelle du globe. Elle suscite des réactions contrastées. Les uns y déchiffrent les promesses d'une planète démocratique unifiée par une culture universelle - une planète réduite par les médias aux dimensions d'un "village global », comme le disait Marshall McLuhan. D'autres y voient la cause d'une inéluctable perte d'identité qu'ils déplorent. Les produits culturels circulent en effet sur l'ensemble de la planète et sont quotidiennement vendus à 6 milliards d'êtres humains, de manière très inégale il est vrai. Les vendeurs de ces produits particuliers - les organisations culturelles - innovent en permanence et font l'objet de remaniements organisationnels constants. Il est illusoire de prétendre en donner une prise de vue instantanée qui fasse droit à leur dynamique. C'est pourquoi nous nous contenterons de dresser un panorama des activités à contenu culturel ainsi que des activités de communication permettant la diffusion de l'information culturelle, en prêtant une attention toute particulière à la répartition mondiale de ces industries que nous inclurons, pour mieux en cerner les enjeux, dans l'économie et la politique culturelle mondiale. L'étude du marché mondialisé de la culture, étant, nous semble-t-il, le préalable nécessaire à tout questionnement sur le devenir du pluralisme culturel.

\section{Présentation des branches d'activités}

\section{Les médias}

2 Les médias principaux de la mondialisation culturelle - cinéma, télévision, livre et presse - constituent de véritables véhicules de brassage culturel ${ }^{1}$. 
3 Ainsi, le cinéma, lorsqu'il produit des films susceptibles d'être exploités hors de leurs milieux d'origine, véhicule des scénarios stéréotypés, produits du marketing, qui collent à l'air du temps des pays occidentaux. Les films américains, suivis de loin par les productions européennes, et quelques rares productions venues de l'Inde, de Taiwan et de Hong-Kong, s'imposent au monde entier. Car, historiquement - la première guerre mondiale ayant propulsé le film américain hors de ses frontières nationales à l'instar de ce médium iconique qu'est le comics - l'image animée se révèle un instrument puissant pour amalgamer les populations immigrantes. Le rayonnement émane toujours d'un centre tutélaire qui impose sa vision du monde aux différentes périphéries ${ }^{2}$. Il s'agit bien, outre « l'accélération extraordinaire des progrès de la rationalité qui brassent et écrasent les cultures locales", de «l'accroissement de la puissance des puissants qui expriment leur point de vue sur le monde, au monde entier $»^{3}$.

4 Cependant, loin de disparaître de la carte du monde, les cultures locales se reformulent, en procédant à un alliage du moderne et de la tradition. En témoigne le formidable brassage de la culture de masse et des cultures populaires dans les produits de l'industrie télévisuelle brésilienne, aujourd'hui l'une des plus performantes du monde et dont les telenovelas atteignent des taux d'audience exceptionnels dans l'Europe septentrionale, centrale et orientale, après avoir tissé un lien transfrontières entre les diverses nations de leur continent d'origine ${ }^{4}$. Livre et édition, quant à eux, résistent toujours aux forces du marché avec des contenus solidaires des langues et des genres littéraires vernaculaires, comme l'indique le tableau suivant:

Vitalité des industries du livre dans le monde $(1996)^{5}$

\begin{tabular}{|l|l|l|l|}
\hline Région ou pays & Population & Nb. de titres publiés par an & Ratio par 100.000 hab. \\
\hline Afrique & $599 \mathrm{M}$ & 7514 & 12 \\
Asie & $3051 \mathrm{M}$ & 220665 & 72 \\
Egypte & $63.3 \mathrm{M}$ & 5925 & 94 \\
France & $58.3 \mathrm{M}$ & 45311 & 777 \\
Pays baltes & $7 \mathrm{M}$ & 6853 & 890 \\
\hline
\end{tabular}

5 Ce qui n'est pas le cas de la presse et la télévision. Partout dans le monde, il existe une demande forte pour des quotidiens, magazines et émissions dans les langues locales et pour des publics singuliers. Or, si la télévision exige la mise en place de supports coûteux que seuls des Etats et sociétés importantes peuvent se permettre, la simplicité des infrastructures de la presse comme de la radio autorise la multiplication des titres et des stations locales. Les médias représentent d'ailleurs un enjeu politique important et s'inscrivent dans tout un environnement social donné. Vecteurs par ailleurs de "conduites modernes", ils sont envisagés comme des agents innovateurs. « Messagers de "la révolution des espérances croissantes", ils propagent les modèles de consommation et d'aspirations symbolisés par les sociétés qui ont déjà accédé à l'étape supérieure de l'évolution. [Ce qui ne fait que] mettre au goût du jour les vieilles conceptions ethnocentriques des théories diffusionnistes du XIX siècle. Le "primitif» est devenu le "sous-développé ", et il n'a comme option que d'imiter les modèles de ses aînés. Cette conception imprègne l'usage des médias audiovisuels mis au service de la « rationalisation» des attitudes des paysans $»^{6}$. Ainsi, dans les années 80, par exemple, 
l'extension agraire au Viêt-Nam a réussi grâce à un soap opera produit par l'université de Can $\mathrm{Tho}^{7}$. Mais les stratégies des industries de la culture ont été profondément remaniées sous l'impact des nouvelles technologies.

\section{Nouvelles technologies et entreprises multimédia}

En effet, avant les années 80 , les industries culturelles étaient spécialisées par filières de supports et de contenus. Mais depuis les années 90, les nouvelles technologies de l'information et de la communication (NTIC) permettent aux industriels de combiner plusieurs médias dans la production des supports et des contenus. Or, dans la lutte contre le sous-développement, la communication devient synonyme de modernisation. Ainsi, la question du développement est définie comme un processus de diffusion de "l'innovation ". Paradoxalement, elles renforcent l'inégalité devant l'information et la culture, puisqu'un dixième de l'humanité au maximum est concerné par ces technologies coûteuses qui confrontent l'individu, pas toujours capable d'en trier et hiérarchiser les contenus, à un véritable déferlement de contenus culturels par le biais de canaux multiples (TV, Internet, télécopie, etc.). D'où l'importance de réfléchir aux enjeux géopolitiques de la communication, et de passer d'une idéologie des systèmes d'information à une problématique de la communication, «admettre que le problème principal, dans ce secteur, n'est pas la production et la diffusion d'un nombre croissant d'informations de toutes natures, et plutôt de reconnaître enfin que ces industries gèrent des visions du monde (...). Si l'information a un prix et devient partout une marchandise lucrative, elle ne se réduit pas à cela, car son usage va au-delà de l'économie "9.

\section{Economie politique mondiale de la culture}

\section{Logiques économiques}

7 C'est la raison pour laquelle nous avons choisi, plutôt que d'étourdir le lecteur de chiffres, de présenter uniquement le rythme d'équipement des ménages en postes TV, qui est un bon indicateur de la perméabilité d'un pays donné aux brassages culturels.

Equipements des ménages en postes de TV en 1985 et 1996 (pour 1000 hab)1011 


\begin{tabular}{|l|l|l|}
\hline Pakistan & 13 & 22 \\
Nigeria & 12 & 38 \\
Inde & 13 & 61 \\
Afrique du Sud & 91 & 101 \\
Viêt-Nam & 33 & 110 \\
Iran & 53 & 140 \\
Indonésie & 38 & 147 \\
Mexique & 113 & 192 \\
Turquie & 159 & 240 \\
Chine & 60 & 250 \\
Brésil & 185 & 279 \\
Corée du Sud & 189 & 323 \\
Russie & 333 & 379 \\
Europe & 600 à 800 & \\
\hline
\end{tabular}

\section{Diversité des situations et inégalités mondiales}

$8 \mathrm{Au}$ vu du panorama mondial du cinéma, du livre, des médias, des nouvelles technologies, et de leur économie, ce qui frappe c'est l'extraordinaire diversité des situations par pays et à l'intérieur de chaque pays.

9 Ceci est renforcé par le fait que la culture industrielle au sens le plus large est très inégalement répartie à la surface du globe. Du côté de la production des biens et des services culturels, les pays industrialisés du triangle Amérique du Nord-Europe-Asie riche exercent une hégémonie qui apparaît dans tous les chiffres. Du côté de la réception des contenus culturels et de la consommation, on retrouve ces mêmes régions en première ligne. Cependant, tous les pays du monde sont gagnés peu ou prou par l'industrialisation de la culture et par les flux mondiaux de marchandises à valeur culturelle, en fonction de leur degré de richesse et de leur développement. Au sein même de ces pays, seules les couches les plus favorisées de la population sont touchées par ces phénomènes et les disparités peuvent être énormes.

10 Le terme de "mondialisation" de la culture est donc impropre si l'on entend par là une répartition des biens culturels industrialisés et marchandisés sur l'ensemble de la planète. En effet, c'est l'extrême inégalité entre pays, et entre catégories sociales à l'intérieur d'un même pays, devant les flux mondiaux de la culture industrialisée, qui est frappante. La mondialisation des flux d'échanges n'est rien, c'est un échange privilégié entre les pays les plus riches. La "mondialisation" de la culture est donc en fait un vaste brassage culturel sous l'emprise hégémonique des industries privées américaines, européennes et asiatiques riches.

11 Or, ce qui est en jeu dans l'hégémonie culturelle et l'emprise privée sur les industries de la culture, c'est la capacité des pays à produire leur propre culture, à la faire perdurer face aux agressions extérieures et à l'invasion sélective des produits culturels. Il en résulte qu'aucune organisation ne peut rester indifférente à l'industrialisation de la culture et que la question des politiques culturelles se pose à tous les échelons des communautés politiques, à l'échelle mondiale. 


\section{Politiques culturelles mondiales} l'économie, leur domaine inclut les médias qui permettent de contrôler plus ou moins la communication culturelle et l'information. Enfin elles transmettent des traditions culturelles s'appuyant sur le patrimoine hérité du passé.

économique, promotion et contrôle de l'information et de la communication, socialisation des individus et transmission du patrimoine culturel et identitaire sont les trois volets de toute politique culturelle. La mondialisation des flux médiatiques et marchands frappe en effet de plein fouet les politiques culturelles des organisations de sorte que, dès l'émergence des médias, s'est posée la question d'une politique mondiale de la culture, désormais prise en charge par des organisations mondiales telles que l'UNESCO ou l'OMC.

\section{L'UNESCO : enjeux et controverses}

14 Depuis sa fondation, l'UNESCO avait élargi son domaine de compétences à une politique mondiale de la culture, militant en faveur de l'idée que la culture est une affaire trop importante pour être abandonnée aux seuls marchands. Mais les produits culturels sont aussi des marchandises, de sorte que dans les années 90 , portées par la vague de libéralisation des échanges, les organisations à vocation commerciale devaient affirmer leur emprise sur les échanges culturels ${ }^{12}$.

\section{Commerce culturel mondial et exception culturelle}

Tombant donc désormais dans le domaine de compétences du GATT et de l'OMC, les produits des industries culturelles ont amené le problème de l'exception culturelle. Or, ses enjeux ne peuvent être évalués qu'à la lumière d'un contexte vaste et mouvant dans lequel le chamboulement géopolitique des années 90 a fait apparaître que l'essor des médias et la croissance économique vont de pair ${ }^{13}$.

Sortir de la logique financière n'est pas à l'ordre du jour. Il est pour le moins stupéfiant que, entre 1992 et 2002, les plus grosses entreprises de communication (Aol-Time-Warner, Disney, Vivendi Universal, etc.) soient aussi devenues les plus puissantes multinationales du monde. C'est ainsi que l'on a vu s'effondrer, ici ou là, des pans entiers des industries culturelles comme du patrimoine. "Aujourd'hui, rares sont ceux qui, au niveau national ou international, s'insurgent contre cette logique de la concentration. D'aucun en arrivent même à dire que la puissance et le pouvoir des groupes de communication sont la garantie de la liberté de l'information $»^{14}$. La concentration des industries culturelles a un second effet pervers : la segmentation du marché en autant de marchés secondaires possibles ayant pour conséquence un effritement du lien social. Or, seuls les Etats sont capables de gérer l'hétérogénéité sociale et culturelle des nations. C'est à eux de maintenir un minimum de cohésion entre les différentes communautés - ce qui, en matière d'industries de la culture et de la communication, se traduit par la problématique de l'intérêt général, l'existence d'un service public, la préservation du caractère national d'une partie des industries culturelles ${ }^{15}$.

Communication et organisation, 26 | 2005 

tenants d'un libéralisme favorable à la segmentation et les partisans d'une problématique plus universaliste liée à la préservation d'une certaine cohésion sociale supposant l'existence d'une régulation pour la garantir.

\section{L'érosion de cultures singulières}

Débat identique chez les ethnologues - spectateurs privilégiés de la mondialisation du marché de la culture - qui témoignent, d'un côté, d'une érosion rapide et irréversible des cultures singulières à l'échelle planétaire et qui observent, de l'autre, que cette érosion est limitée par des éléments solides des cultures-traditions. Il y a en effet, de par le monde, une production culturelle constante, foisonnante, diversifiée, en dépit de l'hégémonie culturelle exercée par les pays industrialisés.

\section{Un véritable ethnocide}

19 Marc Chemilier et Eric de Dampierre ${ }^{16}$, par exemple, constatent que l'art zandé et nzara du chant de la harpe s'est perdu depuis les années 50. En Mélanésie, Annette B. Weiner ${ }^{17}$ a $\mathrm{pu}$ constater que les rites agraires, les échanges cérémoniels et les techniques de construction de canots océaniques observés par Malinowski au début du siècle étaient en grande partie abandonnés, voire tombés dans l'oubli. Enfin, en Asie du sud-est, l'auteur ${ }^{18}$ a constaté la modification, voire la disparition de nombre de rites observés par les Muong et décrits par Jeanne Cuisinier au début du XXe siècle. Ce constat peut être étendu à perte de vue.

Cependant, la diversité des mécanismes d'érosion culturelle défie l'imagination. Si bien qu'aucune explication globale et généralisante ne rend compte des faits. De manière quelque peu arbitraire, disons que de nombreux processus de changements culturels ont affecté les sociétés en venant du dehors. Ainsi, l'expansion mondiale des échanges marchands à partir du XVe siècle a inscrit de nombreuses sociétés à la périphérie du marché mondial ; les conquêtes coloniales, par les Etats-Unis dans l'ouest, la Russie sur son pourtour méridional et oriental, l'Europe partout dans le monde, le Japon en Chine et dans le Pacifique, ont été l'occasion de destruction des cultures singulières à très grande échelle; enfin, la conjonction du nationalisme et de l'économie industrielle et marchande a conféré à l'impérialisme des $\mathrm{XIX}^{\mathrm{e}}$ et $\mathrm{XX}^{\mathrm{e}}$ siècles sa configuration historique singulière et sa violence destructrice. Au nom de la nation dite "civilisée ", il s'agit de transformer le colonisé, de l'atteindre dans sa subjectivité afin de l'arracher à sa prétendue sauvagerie. $\mathrm{Au}$ nom du progrès industriel, il faut mettre l'indigène au travail, contre sa volonté, et le pousser dans l'économie marchande rattachée à la métropole.

Mais, si le militantisme nationaliste et industriel blesse les sociétés de la tradition dans ce qu'elles ont de plus fragile - leur civilisation et l'organisation économique qui lui est subordonnée - jamais les sociétés colonisées n'ont été des jouets passivement voués à la violence du colonisateur. En dépit du choc, elles n'ont pas abdiqué leur initiative et leur inscription dans l'histoire. Elles ont su réinventer les traditions, domestiquer l'apport occidental, se l'approprier et le retourner contre le colonisateur. Elles ont ainsi participé à la production identitaire qui met en échec l'uniformisation par les flux culturels. 

qui se fondent sur deux matrices culturelles, tradition et novation (ou " modernité »), ces deux matrices étant considérées à la fois comme antagonistes entre elles et absolument indispensables. Selon lui, ce sont désormais toutes les populations du monde, sans aucune exception, qui sont tributaires de ces modèles dualistes : « loin de disparaître de la carte $\mathrm{du}$ monde des cultures locales se reformulent, en procédant à un alliage du «moderne " et de la «tradition », et élaborent ainsi les bases de leur propres industries culturelles et de leur propre champ de création artistique $»^{23}$. de la différence. Fernand Braudel ${ }^{24}$ a d'ailleurs souligné combien les civilisations constituent des structures de longue durée qui canalisent le cours de l'histoire. Aussi, la production d'authenticité, les mouvements de relance et les activités de revitalisation révèlent une extraordinaire capacité des cultures à résister à l'érosion. 


\section{Vers le métissage culturel}

Parler de la mondialisation de la culture est un abus de langage. Cette expression, bien commode au demeurant, ne traduit en effet en rien la réalité. Tout au plus peut-on parler de globalisation de certains marchés des biens dits « culturels ».

Les deux débats - celui de l'érosion des cultures singulières et celui de l'américanisation n'en font qu'un. L'humanité, aujourd'hui comme autrefois, reste une machine à fabriquer de la différence, des clivages, du quant à soi, de la distinction de clans, de parlers, de résidences, de classes, de pays, de fractions politiques, des résidences, de classes, d'idéologies ${ }^{25}$, de religions. Ces clivages perpétuent des cultures existantes transmises par tradition, localisées, socialisées, verbalisées, identificatrices et qui remplissent une fonction de boussoles individuelles et collectives. Ces cultures vivent et se transforment. Le marché, moyen d'échange globalisé, mondialise les flux d'objets et de conduites certes, mais dans le même mouvement, approvisionne les sociétés en biens infiniment diversifiés et qui servent à fabriquer de la différence et de l'identité.

Nombreux sont les individus qui doivent maintenant assumer leur devenir au point de rencontre de plusieurs groupes et de plusieurs cultures plus ou moins structurantes. Chaque sujet est de plus en plus le produit de métissages multiples, comme l'indique Armand Mattelart : "L'intensification de la circulation des flux culturels, l'existence bien réelle d'une tendance à la globalisation de la culture ne conduisent pas à l'homogénéisation de la planète, mais vers un monde de plus en plus métissé »`4.

\section{BIBLIOGRAPHIE}

Abdallah-Pretceil, L'éducation interculturelle. Paris : PUF, 1999.

Appadurai, A., Après la colonisation. Les conséquences culturelles de la globalisation. Paris : Payot, 2001.

Balle, F., Médias et sociétés. De Gutenberg à internet. Paris : Montchrestien, 1997.

Basilico, S., La « Viêtcommunication » ou le processus d'insertion des ethnies Thaï et Muong dans l'EtatNation viêtnamien en développement. Thèse de Doctorat d'Anthropologie, Université de Nice SophiaAntipolis, 2001.

Basilico, S., Les objets occidentalisés et la télévision vecteurs de développement du sentiment d'appartenance chez les minorités ethniques du nord Viêt-Nam. Terminal, Paris : l'Harmattan, mars 2002, p.115-125.

Basilico, S., Mondialisation et intégration des minorités ethniques au Viêt-Nam. Paris : l'Harmattan, 2003.

Baudrillard, J., Le système des objets. Paris : Gallimard, 1968.

Baudrillard, J., Pour une critique de l'économie politique du signe. Paris : Gallimard, 1972.

Baudrillard, J., Le crime parfait. Paris : Galilée, 1995. 
Baudrillard, J., L'illusion identitaire. Paris : Fayard, 1996.

Benhamou, F., L'économie de la culture. Paris : La Découverte, 1996.

Bertrand, A., Kalefatides, L., OMC, le pouvoir invisible. Paris : Fayard, 2002.

Braudel, F., Civilisation matérielle, économie et capitalisme : XV-XVIIIe siècles. Paris : Armand Colin, 1967-1975 (3 tomes).

Braudel, F., Grammaire des civilisations. Paris : Arthaud-Flammarion, 1987.

Candau, J., Mémoire et identité. Paris : PUF, 1998.

Chemilier, M., de Dampierre, E., Musique des anciennes cours bancia. Paris : CNRS et Musée de l'Homme, 1996.

Desjeux, D., Taponier, S., Le sens de l'autre. Stratégies, réseaux et cultures en situation interculturelle. Paris : L'Harmattan, 1991.

L'Etat du monde 2001. Paris : La Découverte, 2002.

Gournay, B., Mondialisation et exception culturelle. Paris : Presses de Sciences-Po, 2002.

Gruzinski, S., La pensée métisse. Paris : Fayard, 1999.

Hennebelle, G., Le tribalisme planétaire. Tour du monde des situations ethniques dans 160 pays. Paris : Arléat-Corlet, 1992.

Jeanneney, J-N., Une histoire des médias, des origines à nos jours. Paris : Seuil, 1996.

Lévi-Strauss, C., Identités. Paris : Grasset, 1977.

Lévi-Strauss, C., Race et histoire. Paris : Seuil, 1997.

Mattelart, A., Mondialisation et culture.Universalia 1996, Paris : Encyclopedia Universalis, p.106-111.

Mattelart, A., La Communication-monde, histoire des idées et des stratégies. Paris : La Découverte, 1991.

Mattelart, A., La mondialisation de la communication. Paris : PUF, 2002.

Mattelart T. (Ed.), La mondialisation des médias contre la censure. Paris : De Boeck/INA, 2002.

McLuhan, M., Pour comprendre les médias. Paris : Seuil, 1972.

Poirier, J., Des sociétés traditionnelles aux sociétés postmodernes, une genèse du concept d'identité. In RASSE, P., MIDOl, N., TRIKI, F. (Ed.) Unité-Diversité. Les identités culturelles dans le jeu de la mondialisation. Paris : L'Harmattan, 2002,p.47-67.

Rasse, P., Processus de mondialisation et médiation des identités locales. In Gélerau, M. (Ed.), Médiation des cultures, Paris : Presses Universitaires de Lille, 2000, p.105-115.

Rasse, P., Midol, N., Triki, F. (Ed.), Unité-Diversité. Les identités culturelles dans le jeu de la mondialisation. Paris : L'Harmattan, 2002.

Rasse, P., Les identités locales face au processus de mondialisation. In Rasse, P., Midol, N., Triki, F. (Ed.). Unité-Diversité. Les identités culturelles dans le jeu de la mondialisation. Paris : L'Harmattan, 2002, p.39-49.

Rémeaux, J., Les Empires coloniaux dans le processus de mondialisation. Paris : Maisonneuve, 2002. Senaclens (de), P., La Mondialisation, théories, enjeux, débats. Paris :Armand Colin, 2002.

UNESCO (Ed.), World culture report. Culture, creativity and markets. Paris : UNESCO, 1998. 
UNESCO (Ed.), Déclaration universelle de l'Unesco sur la diversité culturelle. Paris : Flammarion, 1997.

Warnier, J.P., La mondialisation de la culture. Paris : La Découverte, 1999.

Warnier, J.P., Authentifier la marchandise. Anthropologie critique de la quête d'authenticité. Paris :

L'Harmattan, 1996.

Weiner, A.B., Women of value, Men of renown : New Perspectives in Tobriand exchange. St Lucia :

University of Queensmand Press, 1977.

Wolton, D., L'autre mondialisation.Paris : Flammarion, 2003.

Wolton, D., Penser la communication. Paris : Flammarion, 1997

\section{NOTES}

1. Balle, F., Médias et sociétés. De Gutenberg à internet. Paris : Montchrestien, 1997.

2. Mattelart, A., La mondialisation de la communication. Paris : PUF, 2002, p.34-36.

3. Rasse, P., Les identités locales face au processus de mondialisation. In Rasse, P., Midol, N., Triki, F. (Ed.). Unité-Diversité. Les identités culturelles dans le jeu de la mondialisation. Paris: L'Harmattan, 2002, p.39.

4. Mattelart, A., Mondialisation et culture. In Universalia 1996. Paris : Encyclopedia Universalis, p.106-111

5. L'Etat du monde, op.cit.

6. Mattelart, A., La mondialisation de la communication. op.cit., p.62.

7. Basilico, S., Mondialisation et intégration des minorités ethniques au Viêt-Nam. Paris : l'Harmattan, 2003, p.82.

8. Mattelart, A., La mondialisation de la communication. op.cit., p.61.

9. Wolton, D., L'autre mondialisation. Paris : Flammarion, 2003, p.36.

10. L'Etat du monde, op. cit.

11.

12. Warnier, J-P., La mondialisation de la culture. Paris : La Découverte, 1999, p.74.

13. ibid., p.76.

14. Wolton, op. cit. p. 37.

15. Ibid.

16. Chemilier, M., de Dampierre, E., Musique des anciennes cours bancia. Paris : CNRS et Musée de l'Homme, 1996.

17. Weiner, A-B., Women of value, Men of renown: New Perspectives in Tobriand exchange. St Lucia : University of Queensmand Press, 1977.

18. Basilico, S., Les objets occidentalisés et la télévision vecteurs de développement du sentiment d'appartenance chez les minorités ethniques du nord Viêt-Nam. Terminal, Paris : l'Harmattan, mars 2002.

19. Basilico, S., La «Viêtcommunication » ou le processus d'insertion des ethnies Thaï et Muong dans l'Etat-Nation viêtnamien en développement. Thèse de Doctorat d'Anthropologie, Université de Nice Sophia-Antipolis, 2001.

20. Baudrillard, J., Le crime parfait. Paris : Galilée, 1995.

21. C'est le cas notamment de Jean-Pierre Warnier, de Jean Poirier et de nombreux ethnologues.

22. L'auteur définit l'hétéro culture comme les « 2 grandes matrices qui s'imposent à chaque culture, à chaque individu, c'est-à-dire d'une part la tradition (la récurrence, la fidélité à la coutume presque toujours sacralisée) et la "modernité" (le changement), qui est souvent perçu en termes de valeurs ». POIRIER, J., Des sociétés traditionnelles aux sociétés postmodernes, une 
genèse du concept d'identité. In RASSE, P., MIDOl, N., TRIKI, F. (Ed.) Unité-Diversité. Les identités culturelles dans le jeu de la mondialisation, op.cit., p.47-67.

23. Mattelart, A., La mondialisation de la communication, op.cit., p.107.

24. Braudel, F., Grammaire des civilisations, Paris : Arthaud-Flammarion, 1987.

25. Mattelart, A., La mondialisation de la communication, op.cit., p.106.

\section{NOTES DE FIN}

i.

\section{RÉSUMÉS}

Ce qui frappe lors de l'évocation des organisations de la culture - des industries de l'information et de la culture aux organismes internationaux tels l'UNESCO ou l'OMC - c'est l'extrême diversité des situations, l'émission et la production de services culturels se faisant, schématiquement, sur un unique axe nord-sud. Aujourd'hui, ce qui est en jeu dans l'hégémonie culturelle et l'emprise privée sur les industries de la culture, c'est la capacité des pays à produire leur propre culture et la propension des peuples à résister à l'érosion, de sorte qu'on ne peut parler de "mondialisation » si l'on entend par là une répartition des biens culturels industrialisés et marchandisés sur l'ensemble de la planète ou un ethnocide généralisé.

We can first notice that roughly speaking, cultural production and broadcasting are primarily directed along a North-South axis . Bearing this in mind, one is struck by the extremely wide range of situations related to cultural organisations - including the industries commodifying media and culture as well as international organisations, such as UNESCO or the WTO. Nowadays, what is at stake, is countries' capacity to produce their own culture and to resist cultural erosion, so much so that "globalisation" is not a proper term if it's defined as "industrialized cultural goods repartition" or "generalized ethnocide".

\section{AUTEUR}

\section{SANDRINE BASILICO}

Docteur en Anthropologie de la Communication Sociale, l'auteur est chercheur au LAMIC (Université de Nice). Sa thématique de recherche - la mondialisation - s'effectue sur l'aire asiatique comme en témoignent ses différentes publications, dont la mondialisation et intégration des minorités ethniques au Viêt-Nam, L'Harmattan, 2003, les objets occidentalisés et la télévision vecteurs du développement du sentiment d'appartenance des ethnies Thaï et Muong, Terminal, L'Harmattan, 2002 et la mondialisation source de modification de l'identité sociale et culturelle des Thaï et des Muong, CIFSIC, 2003. Mail : sbasilico@free.fr 